\title{
Effect of Gallic Acid on Dementia Type of Alzheimer Disease in Rats: Electrophysiological and Histological Studies
}

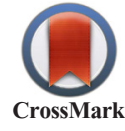

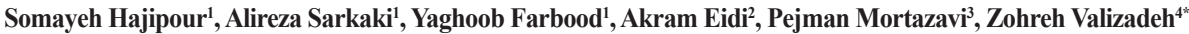 \\ 1. Physiology Research Center, Department of Physiology, School of Medicine, Ahvaz Jundishapur University of Medical Sciences, Ahvaz, Iran \\ 2. Department of Biology, Science and Research Branch, Islamic Azad University, Tehran, Iran. \\ 3. Department of Pathology, Faculty of Specialized Veterinary Science, Science \& Research Branch, Islamic Azad University, Tehran, Iran. \\ 4. Department of Nursing and Midwifery, Dezfoul Branch, Islamic Azad University, Dezfoul, Iran.
}

Citation: Hajipour, S., Sarkaki, A. R., Farbood, Y., Eidi, A., Mortazavi, P., \& Valizadeh, Z. (2016). Effect of gallic acid on dementia type of alzheimer disease in rats: Electrophysiological and histological studies. Basic and Clinical Neuroscience, 7(2), 97-106. http://dx.doi.org/10.15412/J.BCN.03070203

http://dx.doi.org/10.15412/J.BCN.03070203

Article info:

Received: 24 April 2015

First Revision: 25 July 2015

Accepted: 01 September 2015

Key Words:

Alzheimer disease,

Beta-amyloid, Long-term

potentiation, Neuronal

apoptosis, Rat

\begin{abstract}
A B S T RA C T
Introduction: To study the effect of gallic acid (GA) on hippocampal long-term potentiation (LTP) and histological changes in animal model of Alzheimer disease (AD) induced by betaamyloid $(\mathrm{A} \beta)$.

Methods: Sixty-four adult male Wistar rats $(300 \pm 20$ g) were divided into 8 groups: 1) Control (Cont); 2) AD; 3) Sham; 4-7) AD+GA (50, 100, and $200 \mathrm{mg} / \mathrm{kg}$ for 10 days, orally) or vehicle, 8) Cont $+\mathrm{GA} 100, \mathrm{~A} \beta(1 \mu \mathrm{g} / \mu \mathrm{L}$ in each site) was infused into hippocampus bilaterally. Changes of amplitude and slope of LTP induced in hippocampal dentate gyrus (DG) were evaluated by high frequency stimulation (HFS) of perforant path (PP).

Results: Data showed that LTP amplitude and area under curve significantly impaired in AD rats $(\mathrm{P}<0.001)$, while significantly improved in $\mathrm{AD}$ rats treated with $\mathrm{GA}(\mathrm{P}<0.05, \mathrm{P}<0.01)$.

Conclusion: Current findings suggest that GA reduces neural damage and brain amyloid neuropathology and improves cognitive function via free radicals scavenging and inhibiting oligomerization of $\mathrm{A} \beta$ but with no effect on healthy rats.
\end{abstract}

\section{Introduction}

lzheimer disease $(\mathrm{AD})$ is the most com1 mon neurodegenerative disorder affecting the elderly with a progressive cognitive decline and memory loss (dementia). Multiple pathogenetic factors, including aggregated beta-amyloid $(A \beta)$, neurofibrillary tangles (NFTs), cholinergic dysfunction, and oxidative stress are involved in AD (Babri et al., 2014). The reasons for $\mathrm{A} \beta$ deposition are not clear, but an impaired clearance of $\mathrm{A} \beta$ at the blood-brain barrier may be implicated and oxidative stress possibly plays a major role in this process (Ehrlich, Hochstrasser, \& Humpel, 2013). To date, no effective treatments to prevent or slow dementia are available (Scuderi et al., 2014).

Aging, the major risk factor for $\mathrm{AD}$, leads to the loss of free radical scavenging ability by endogenous mechanisms (Nobakht et al., 2011). As one of the most important hallmarks of $\mathrm{AD}, \mathrm{A} \beta$ plays important roles in inducing reactive oxygen species (ROS) generation, mitochondrial

\footnotetext{
* Corresponding Author:

Zohreh Valizadeh, $\mathrm{PhD}$

Address: Department of Nursing and Midwifery, Dezfoul Branch, Islamic Azad University, Dezfoul, Iran.

Tel:+98 (916) 3051938

Fax: +98 (611) 3738248
}

E-mail: valizadeh_z@yahoo.com. 
dysfunction, and apoptotic cell death in neurons (Sun et al., 2014b). Although the etiology of AD is largely unknown, it has been hypothesized that multiple factors, including genetic factors, oxidative stress, intracellular and or extracellular accumulation of $\mathrm{A} \beta$, excitotoxicity, inflammation, mitochondrial dysfunction, alteration of cytoskeleton, synapse components, and neuronal loss may play essential roles in the onset of the disease (Ferreiro et al., 2012).

Oxidative stress (OS) and inflammation are thought to be the major factors in brain aging and age-related neurodegenerative diseases (Engelhart et al., 2002; ShukittHale, Carey, Simon, Mark, \& Joseph, 2006). Humans and animals show increased motor and cognitive declines with aging, that are thought to be due to increased susceptibility to the long-term effects of OS and inflammation (Joseph, Shukitt-Hale, \& Casadesus, 2005; Zhang et al., 2013). Foods containing high levels of antioxidants may also slow the progression of $\mathrm{AD}$, possibly by avoiding or neutralizing the damaging effects of free radicals (Kostrzewa \& Segura-Aguilar, 2003; Hartman et al., 2006).

Synaptic pathology is considered a major and early contributor to the cognitive deficits and reduced cerebral activity of AD (Kim, Anwyl, Suh, Djamgoz, \& Rowan, $2001)$. In addition to their neurotoxic role in $A D, A \beta$ peptides are also known to play physiological roles. Low concentrations of $A \beta_{1-40}$ play a role in regulating cerebellar granule neurons (CGNs) maturation through the p75 neurotrophin receptor (p75 (NTR)) (Zhan, Yao, Liu, Ma, $\&$ Mei, 2014).

On the other hand, the role of $\beta$-APP-related amyloidogenic peptides in mediating synaptic disruption has been examined by studying their direct effects on synaptic mechanisms, especially long-term potentiation (LTP). LTP is a neurophysiological model of activity dependent changes in synaptic strength that is believed to underlie information storage (Kim et al., 2001) and memory as one of the basic cognitive functions (Sladjana, 2011). Extensive research about the potential therapeutic effects of antioxidants in the treatment of $\mathrm{AD}$ has produced promising results. Antioxidants such as gallic acid (GA) and other polyphenols have been found to improve cognitive functions in aged rats and prevent learning and memory deficits following intracerebroventricular (ICV) infusion of $A \beta$ (McDaid et al., 2005).

GA, an endogenous product found in plants as a phenolic compound, is a natural product used in chemical industries such as dye making and tanning of leather
(Qi, Jing, Wang, \& Zhan, 2009). Also it has a broad assortment on biological activities such as antioxidant and anti-inflammatory processes (Kratz et al., 2008b). GA as a form of gallate, is generally used as antioxidants by food supplements and pharmaceutical companies (Kratz et al., 2008a).

GA plays a neuroprotective role through involving the antioxidant and inflammation pathways in the animal models of neurodegenerative diseases (Mansouri et al., 2013; Korani, Farbood, Sarkaki, Fathi-Moghaddam, \& Mansouri, 2014; Naghizadeh \& Mansouri, 2014). GA has a possible protective effect against neurotoxicity due to NMDA receptors sensitivity and excitotoxicity induced by glutamate after cerebral ischemia that followed by $\mathrm{Ca}^{+2}$ influx and thereby intracellular $\mathrm{Ca}^{+2}$ accumulation induced neuronal apoptosis. On the other hand, GA with its antioxidative effect may oppose the NMDA receptors activation and thereby has a protective effect on neurotoxicity and or excitotoxicity following brain injury (Korani et al., 2014). GA binds to proteins and key minerals such as iron, zinc, calcium and affects their bioavailability by forming insoluble complexes (Niho et al., 2001). So this study aimed to investigate the effects of oral administration of GA on LTP recorded from DG of hippocampus and histological changes in the rat model of AD (by intrahippocampal injection of $A \beta_{1-42}$ ).

\section{Materials \& Methods}

\subsection{Animals}

Sixty-four adult male Wistar rats $(300 \pm 20 \mathrm{~g})$ obtained from central animal house of Ahvaz Jundishapur University of Medical Sciences (AJUMS, Ahvaz-Iran) were used in this study. They were housed individually in standard cages under controlled room temperature $\left(20 \pm 2^{\circ} \mathrm{C}\right)$, humidity (50\%-55\%) and light exposure of 12:12h light/ dark cycle with free access to food and water ad libitum. All experiments were carried out during the light phase of the cycle (8:00 AM to 5:00 PM). Animal handling and experimental procedures were performed under observance of the University and Institutional legislation, controlled by the Local Ethics Committee for the Purpose of Control and Supervision of Experiments on Laboratory Animals. The animals divided randomly into 8 groups (8 rats in each) as following: (1) Control (intact); Without any operation and treatment; (2) Sham operated (Sham); Rats received $1 \mu \mathrm{L}$ normal saline into hippocampus bilaterally under operation and received $5 \mathrm{~mL}$ normal saline orally for 10 days postoperatively; (3) AD received $1 \mu \mathrm{g} / \mu \mathrm{L} \mathrm{A} \beta_{1-42}$ into hippocampal CA1 area bilaterally without any treatment, (4-6) $\mathrm{AD}+\mathrm{GA}$ groups, $\mathrm{AD}$ rats re- 
ceived 50, 100, $200 \mathrm{mg} / \mathrm{kg} / 5 \mathrm{~mL}$ GA by oral gavage for 10 consecutive days from the second day of operation, (7) $\mathrm{AD}+\mathrm{Veh}$; $\mathrm{AD}$ rats received the same volume of normal saline orally for the same period), and (8) Positive control (Control+GA100); Healthy rats received most effective dose of GA determined in AD rats $(100 \mathrm{mg} / \mathrm{kg})$ by oral gavages for 10 consecutive days.

\subsection{Beta-amyloid 1-42 $\left(\mathrm{A} \beta_{1-42}\right)$}

Beta-amyloid powder (Sigma-Aldrich Co., USA) was dissolved in normal saline (PH 7.2) at the concentration of $1 \mu \mathrm{g} / \mu \mathrm{L}$ and solution was incubated at $37^{\circ} \mathrm{C}$ for one week before application. The drug was injected into hippocampal CA1 areas of rats' brain bilaterally $(1 \mu \mathrm{g} / 1 \mu \mathrm{L}$ to each side) to induce the early stage of AD (Kim et al., 2001; Goryacheva et al., 2010).

\subsection{Preparation of gallic acid}

Gallic acid (Sigma-Aldrich Co., USA, $\mathrm{LD}_{50}=5000 \mathrm{mg}$ / $\mathrm{kg}$ and $\mathrm{ED}_{50}=5-10 \mu$ moles) was dissolved in normal saline, and administered $(50,100,200 \mathrm{mg} / \mathrm{kg} / 5 \mathrm{~mL})$ to rats orally for 10 days after AD induction (Ferruzzi et al., 2009).

\subsection{Surgery and drug administration}

Animals were anesthetized with intraperitoneal injection of $90 \mathrm{mg} / \mathrm{kg}$ ketamine $\mathrm{HCl}$ and $10 \mathrm{mg} / \mathrm{kg}$ xylazine (Alfasan, Woerden-Holland). In brief, after anesthesia, animals' head was fixed in a stereotaxic apparatus (Narishige, Tokyo, Japan) and $\mathrm{A} \beta_{1-42}(1 \mu \mathrm{g} / \mu \mathrm{L}$ in each site) was infused into the hippocampal CA 1 area bilaterally at a rate of $1 \mu \mathrm{L} / 5 \mathrm{~min}$ with using a $10-\mu \mathrm{L}$ Hamilton syringe connected to an infusion pump (WPI 101i, USA), after infusion, the cannula was left in place for additional 3 minutes to allow the complete diffusion of drug (Wen et al., 2014). Infusion was done with coordinates of $\mathrm{AP}=-4.8 \mathrm{~mm}$ from the bregma, $\mathrm{ML}= \pm 3.5 \mathrm{~mm}, \mathrm{DV}=-4 \mathrm{~mm}$ from dura mater, according to the Paxinos and Watson rat brain atlas for stereotaxic surgery (Paxino \& Wastene, 2006). All animals allowed the recovery period before testing (7-10 days) while the gavages of GA were started from the second day after operation (Ferihan \& Sibel, 2006).

\subsection{Electrophysiological recording}

Under chloral hydrate anesthesia (300 and $400 \mathrm{mg} / \mathrm{kg}$ ), a pair of recording tungsten wire microelectrodes (CFW, USA) was implanted into the left dentate gyrus (DG) at $\mathrm{AP}=-3.8 \mathrm{~mm}$ from bregma, $\mathrm{ML}=-2.2 \mathrm{~mm}$, and $\mathrm{DV}=3.5$ $\mathrm{mm}$ from dura. A pair of stimulating stainless steel wire microelectrodes (CFW, USA) was implanted into ipsilateral perforant path $(\mathrm{PP})$ at $\mathrm{AP}=-7.5 \mathrm{~mm}$ from bregma, $\mathrm{ML}=-4 \mathrm{~mm}$, and DV=3.9 $\mathrm{mm}$ from the dura. Single monopolar pulses (duration $50 \mu \mathrm{s}$ ) were delivered at 30 -second intervals. The baseline intensity was selected to result in field excitatory post-synaptic potential (fEPSP) with $40 \%$ of its maximum amplitude while $80 \%$ of its maximum amplitude was selected High-frequency stimulation (HFS) by input/output (I/O) curve with different intensities. The signal was amplified $(\times 1000)$, filtered $(0.1 \mathrm{~Hz}-3$ $\mathrm{kHz}$ ), digitized at $2 \mathrm{kHz}$, and saved in the computer. To induce LTP, high-frequency stimulation consisted of 6 trains of 6 pulses $(50 \mu \mathrm{s})$ at $400 \mathrm{~Hz}$, with $100 \mathrm{~ms}$ between each train, repeated 6 times at a 20 -second interval (Gureviciene et al., 2004). In vivo, LTP was recorded at 1, 3, 24, and 48 hours after HFS with weak anesthesia. Amplitude (Amp) and area under curve (AUC) of population spikes (PSs) were measured. The recorded PS was analyzed as the percentage increase of baseline fEPSP.

\subsection{Histological evaluation}

At the end of the tests, in order to evaluate the histological changes of brain hippocampus, animals were anaesthetized deeply with overdose of ketamine $\mathrm{HCl}$ and killed. Then, their brains were removed, fixed in $4 \%$ paraformaldehyde and dissected into hippocampal blocks. Following routine processing in paraffin, serial coronal sections of the brain were cut at $8-\mu \mathrm{m}$ thickness in a rotary microtome (Leitz, 1512, Germany). The parts of each brain section were stained with modified Bielschowsky staining.

This staining gives a good compromise between sensitivity for plaques and tangles and can be used as a single stain for diagnosis of AD. Briefly, sections were deparaffinized by xylene and alcohols into tap water before being placed into fresh $20 \%$ silver nitrate solution for $20 \mathrm{~min}$ utes. After washing thoroughly with distilled water, slides were immersed in $20 \%$ silver nitrate solution titrated with fresh sodium hydroxide and evaporated ammonia (200 $\mathrm{mL}$ of $28 \%$ ammonium hydroxide by leaving in an open beaker for $20 \mathrm{~min}$ in a fume cupboard). After 15 minutes, slides were washed with ammonia water before being individually developed with $100 \mathrm{~mL}$ of developer $(20 \mathrm{ml}$ of formaldehyde, $100 \mathrm{~mL}$ distilled water, $20 \mu \mathrm{L}$ concentrated nitric acid, and $0.5 \mathrm{~g}$ citric acid). Then, they were added to $50 \mathrm{~mL}$ of titrated silver nitrate solution. Slides were rinsed in tap water, fixed in 5\% sodium thiosulfate, and dehydrated through alcohols and xylene. Congo red staining is an accepted histochemical marker for the Aßpleated-sheet structure of amyloid. Sections were deparaffinized through xylene and alcohols into tap water. 
Afterwards, slides were immersed in alkaline sodium chloride. Twenty minutes later, they were immersed in alkaline Congo red solution for 20 minutes and then differentiated with alcoholic potassium. Next, slides were counterstained with alum hematoxylin and dehydrated through alcohols and xylene. Measurement of the area was performed using the OLYSIA BioReport software imaging system (Olympus Corporation, Tokyo, Japan) (Kowall, Beal, Busciglio, Duffy, \& Yankner, 1991) and neuritic plaques were counted on modified Bielschowsky stained separately in hippocampus at $100 \times$ magnification.

\subsection{Statistical analysis}

Data were expressed as mean \pm S.E.M. The data of fEPSP slope, population spike Amp and AUC in different groups were analyzed using repeated measures ANOVA followed by Tukey post hoc test. $\mathrm{P}$ value less than 0.05 was considered significant.

\section{Results}

\subsection{Electrophysiology}

Examples of LTP recorded from DG are shown in Figure 1. Animals were subjected to HFS and displayed LTP traces. Oral administration of GA $(50,100$, and 200 $\mathrm{mg} / \mathrm{kg}$ ) for 10 days in AD rats significantly increased the PS Amp (Figure 2). At 1, 3, 24, 48 hours after HFS, the mean amplitude values did not indicate any differ- ences between control $(118.76 \pm 15.18,130.27 \pm 17.87$, $127.97 \pm 18.03$, and $131.63 \pm 16.12$, respectively) and sham groups (123.69 $\pm 18.06,112.44 \pm 9.38,120.85 \pm 16.95$, and $123.31 \pm 14.84$, respectively). Figure $2 \mathrm{~A}$ shows significantly decreased PS Amp of AD group (79.11 \pm 6.57 , $63.21 \pm 10.02$, 56.29 \pm 9.15 , and 59.1 \pm 4.89 , respectively) compared to sham group ( $\mathrm{P}<0.05, \mathrm{P}<0.01$, for $\mathrm{AD}$ vs. Sham). On the other hand, at 1, 3, 24, 48 hours after HFS, the mean amplitude values were not different between $\mathrm{AD}$ and $\mathrm{AD}+$ Veh groups $(79.11 \pm 6.57,63.21 \pm 10.02$, $56.29 \pm 9.15$, and $59.1 \pm 4.89$, respectively) groups, but increased significantly in AD+GA group $(100,200)$ at 1 , 3, 24, 48 hours after HFS $(149.58 \pm 20.54,155.74 \pm 22.48$, $136.46 \pm 21.27$, and $122.05 \pm 11.1$, respectively $(\mathrm{P}<0.001$, $\mathrm{P}<0.01, \mathrm{P}<0.05 \mathrm{AD}+\mathrm{GA}$ vs. AD+Veh, Figure $2 \mathrm{~A}-\mathrm{D})$.

As illustrated in Figure 3, comparing the percentage of $\mathrm{AUC}$, there were no difference between the control and sham groups at 1, 3, 24, and 48 hours after HFS $(121.91 \pm 22.29,115.94 \pm 27.27,127.27 \pm 16.71$, and $121.24 \pm 11.12$, respectively) while it decreased significantly at the same times in $\mathrm{AD}+\mathrm{Veh}(77.25 \pm 7.93,62.68 \pm 11.5$, $29.26 \pm 8.46$, and $35.74 \pm 10.1$, respectively) compared to sham group $(* \mathrm{P}<0.05, * * \mathrm{P}<0.01$, Figure $3 \mathrm{~A})$.

Figure $3(\mathrm{~B}, \mathrm{C}$, and $\mathrm{D})$ shows that $\mathrm{AUC}$ for $\mathrm{AD}$ and $\mathrm{AD}+$ Veh groups decreased significantly compared to sham group, while it reversed in $\mathrm{AD}+\mathrm{GA}(50,100,200)$ groups significantly (for $100 \mathrm{mg} / \mathrm{kg}$ as best effective dose $155.57 \pm 16.12,150.48 \pm 31.6,137.27 \pm 16.46$, and

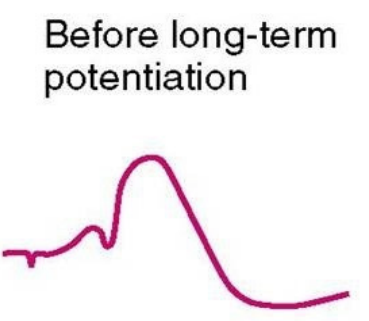

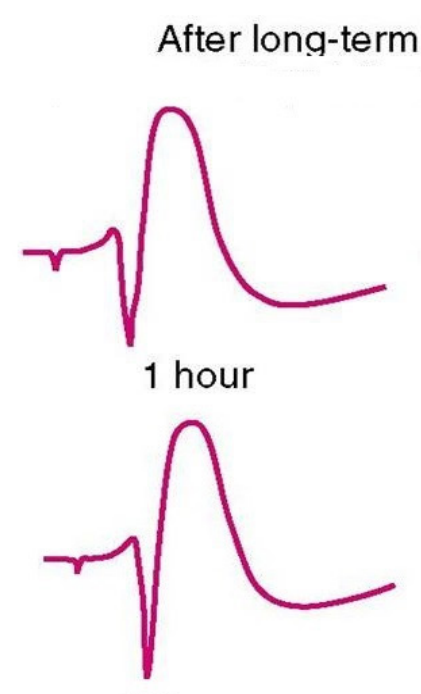

24hours

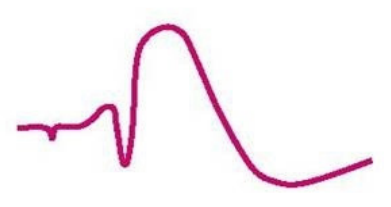

3 hours

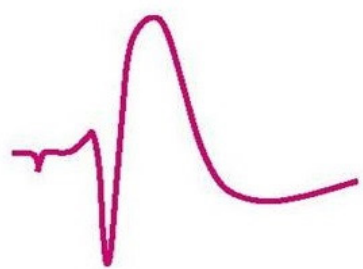

48 hours

NEUR SCIENCE

Figure 1. Recording of fEPSP before and after LTP induction in different groups. Population spikes were recorded from hippocampal dentate gyrus at 1 hour before and 1,3,24, and 48 hours after high frequency stimulation to brain perforant path. 
102.23 \pm 12.6 , respectively $* \mathrm{P}<0.05, * * \mathrm{P}<0.01 \mathrm{AD}+\mathrm{GA}$ vs. AD+Veh, Figure 3B, C, D).

As shown in Figure 4 (A and B), administration of effective dose of GA $(100 \mathrm{mg} / \mathrm{kg})$ to control animals had no significant effects on electrophysiological properties of hippocampus (PSs Amp and AUC) compared to control group while in contrast to $\mathrm{AD}$ groups, this dose of GA improved amplitude and AUC of LTP significantly (Figures $2 \mathrm{C}$ and $3 \mathrm{C}$, respectively, $* \mathrm{P}<0.05$, $* * \mathrm{P}<0.01$, $* * * \mathrm{P}<0.001)$.

\subsection{Histological evaluation}

In this study by using the Bielschowsky silver staining, diffuse plaques were detected in the hippocampus. As shown in Figure 5 E and D, treatment with GA improved the histological damage (removed the AD plaques in CA1 region of Hippocampus) compared to $\mathrm{B}$ and $\mathrm{F}$ sections from $\mathrm{AD}$ and $\mathrm{AD}+\mathrm{Veh}$ groups.

\section{Discussion}

Our findings showed that intrahippocampal infusion of beta-amyloid induced rat model of AD with formation the amyloid plaques as well as long-term potentiation impairment in hippocampus. Beta-amyloid (A $\beta$ ) is a small peptide that plays a potent useful role in synaptic plasticity as well as forming amyloid plaques in AD. Recent studies suggest that $A \beta$ deposition is deleterious not only in $\mathrm{AD}$, but also in Parkinson disease (PD), and depression. This $A \beta$ effect is associated with inflammatory processes (Hochstrasser, Hohsfield, Sperner-Unterweger, \& Humpel, 2013)

Oxidative stress, mitochondrial and energy metabolism dysfunction, excitotoxicity, inflammation, and apoptosis have been recognized as influential factors in $\mathrm{AD}$ (Chen et al., 2014). Studies suggested that $A \beta$ accumulation in the brain may impair glucose homeostasis in the brain and peripheral tissues. So, cognitive dysfunction attributable to $A \beta$ accumulation in the hippocampus might be related
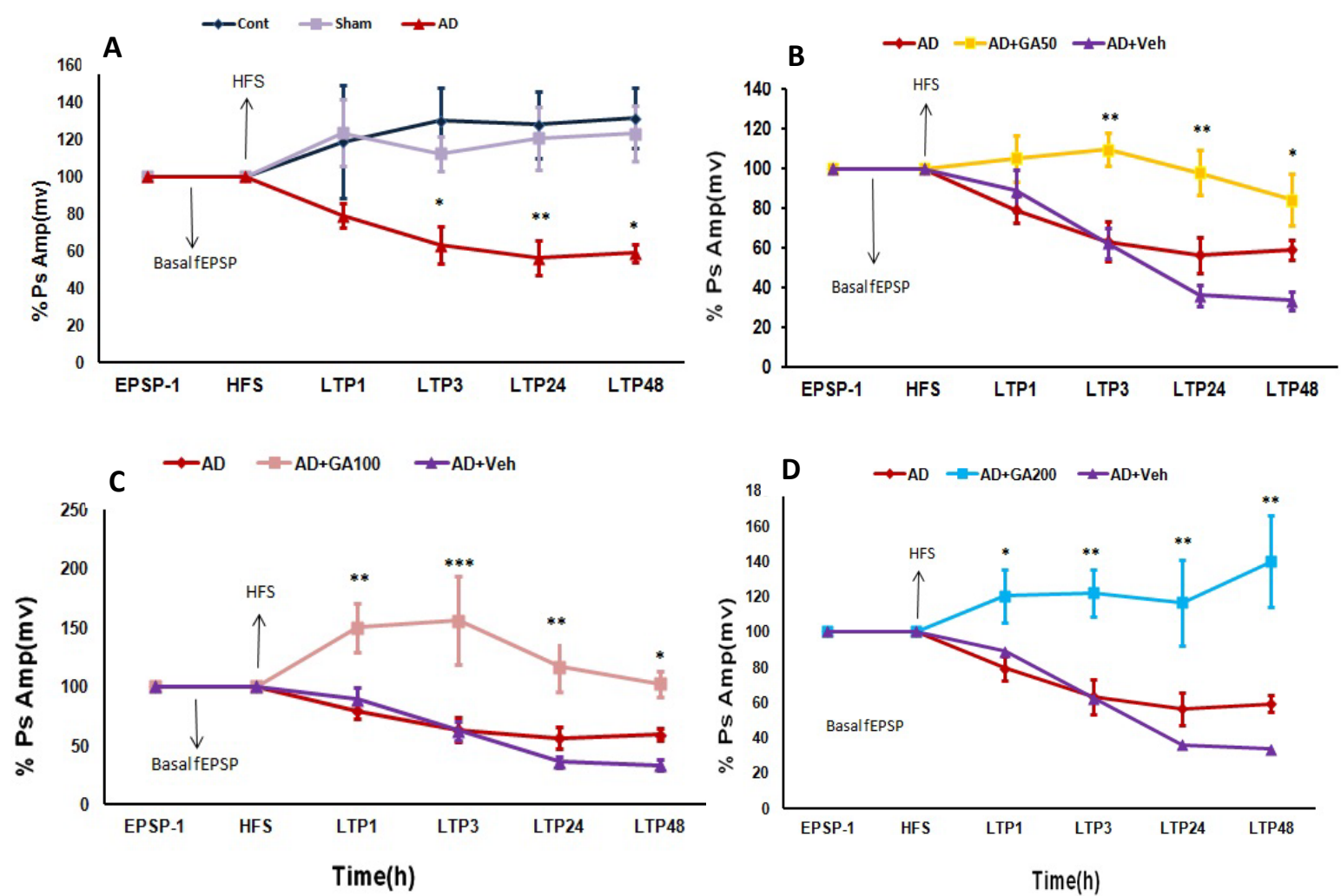

NEUR:SCIENCE

Figure 2. Percentages of population spikes amplitude in different groups during basal fEPSP and LTP recorded from hippocampal dentate gyrus at one hour before and 1,3,24, and 48 hours after high frequency stimulation to brain perforant. Repeated measures 2-way ANOVA, followed by HSD post hoc test $(n=8)$. There were no significant differences between control and sham groups (A). Amplitude was reduced significantly in AD group at 1, 3, 24, and 48 hours after HFS compared to sham group $\left({ }^{*} \mathrm{P}<0.05, * * \mathrm{P}<0.01\right.$ vs. Sham). Amplitude was increased significantly in $\mathrm{AD}+\mathrm{GA}$ groups $\left(\mathrm{B}, \mathrm{C}, \mathrm{D},{ }^{*} \mathrm{P}<0.05,{ }^{* *} \mathrm{P}<0.01\right.$, and $* * * \mathrm{P}<0.001$ for $\mathrm{AD}+\mathrm{GA}$ vs. $\mathrm{AD}+\mathrm{Veh})$. 

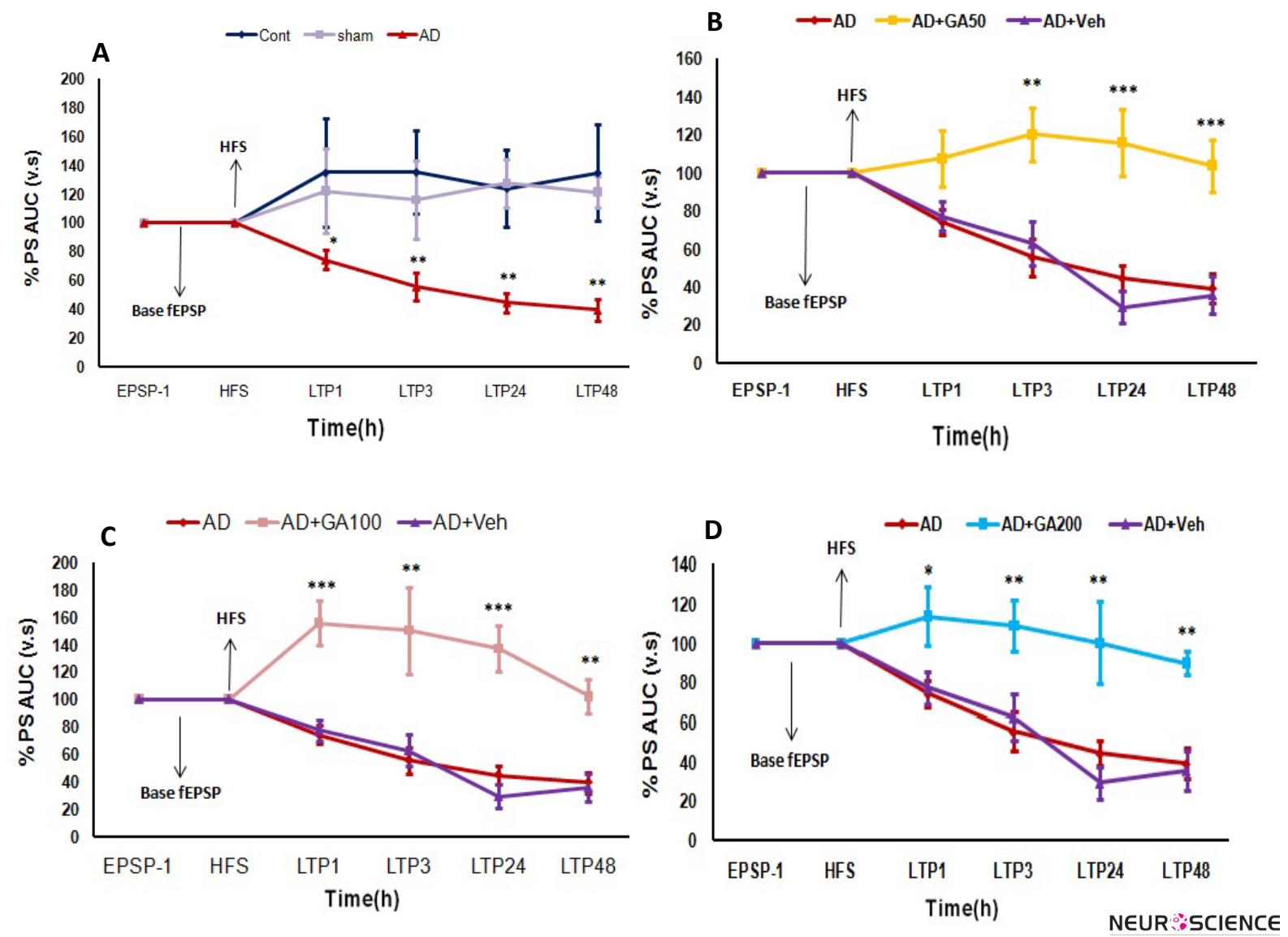

Figure 3. Percentages of population spikes AUC in different groups during basal fEPSP and LTP recorded from hippocampal DG at one hour before and 1, 3, 24, $48 \mathrm{~h}$ after HFS to brain PP. Repeated measures 2-way ANOVA, followed by HSD post hoc test $(n=8)$. There are no significant differences between control and sham groups (A). AUC was reduced significantly in AD group at $1,3,24,48$ hours after HFS $\left({ }^{*} \mathrm{P}<0.05,{ }^{*} \mathrm{P}<0.01 \mathrm{vs}\right.$. Sham). AUC was increased significantly in AD+GA groups $(\mathrm{B}, \mathrm{C}, \mathrm{D}$, ${ }^{*} \mathrm{P}<0.05,{ }^{* * \mathrm{P}}<0.01, * * * \mathrm{P}<0.001$ vs. $\left.\mathrm{AD}+\mathrm{Veh}\right)$.
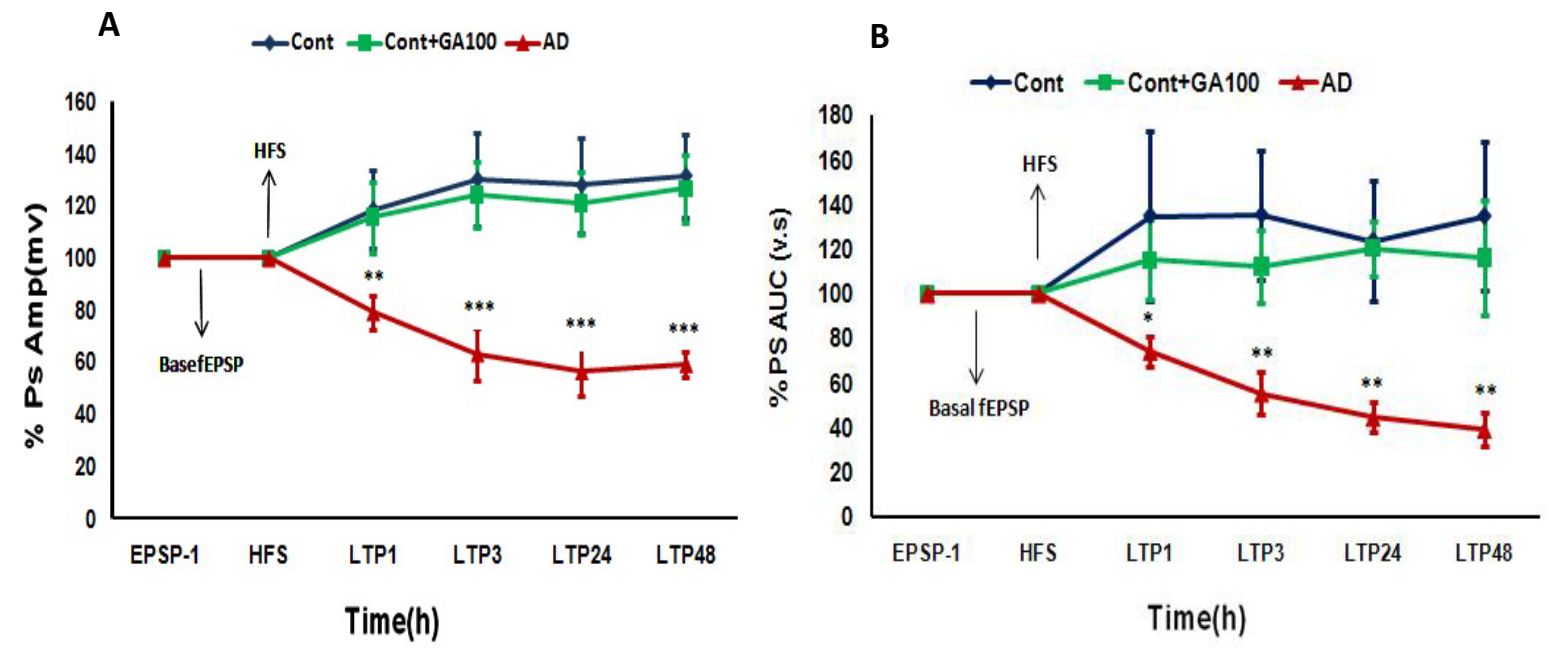

Figure 4. Percentages of population spikes, AMP, and AUC in Control, Cont+GA100, and AD groups during basal fEPSP and LTP recorded from hippocampal DG at 1 hour before and 1, 3, 24, and $48 \mathrm{~h}$ after HFS to brain PP. Repeated measure ANOVA, followed by HSD post hoc test $(n=8)$. There are no significant differences between control and Cont $+G A 100$ groups (A and B). AMP and AUC were reduced significantly in AD group during same times after $\mathrm{HFS}\left({ }^{*} \mathrm{P}<0.05,{ }^{* *} \mathrm{P}<0.01,{ }^{* * *} \mathrm{P}<0.001\right.$ vs. control groups). 

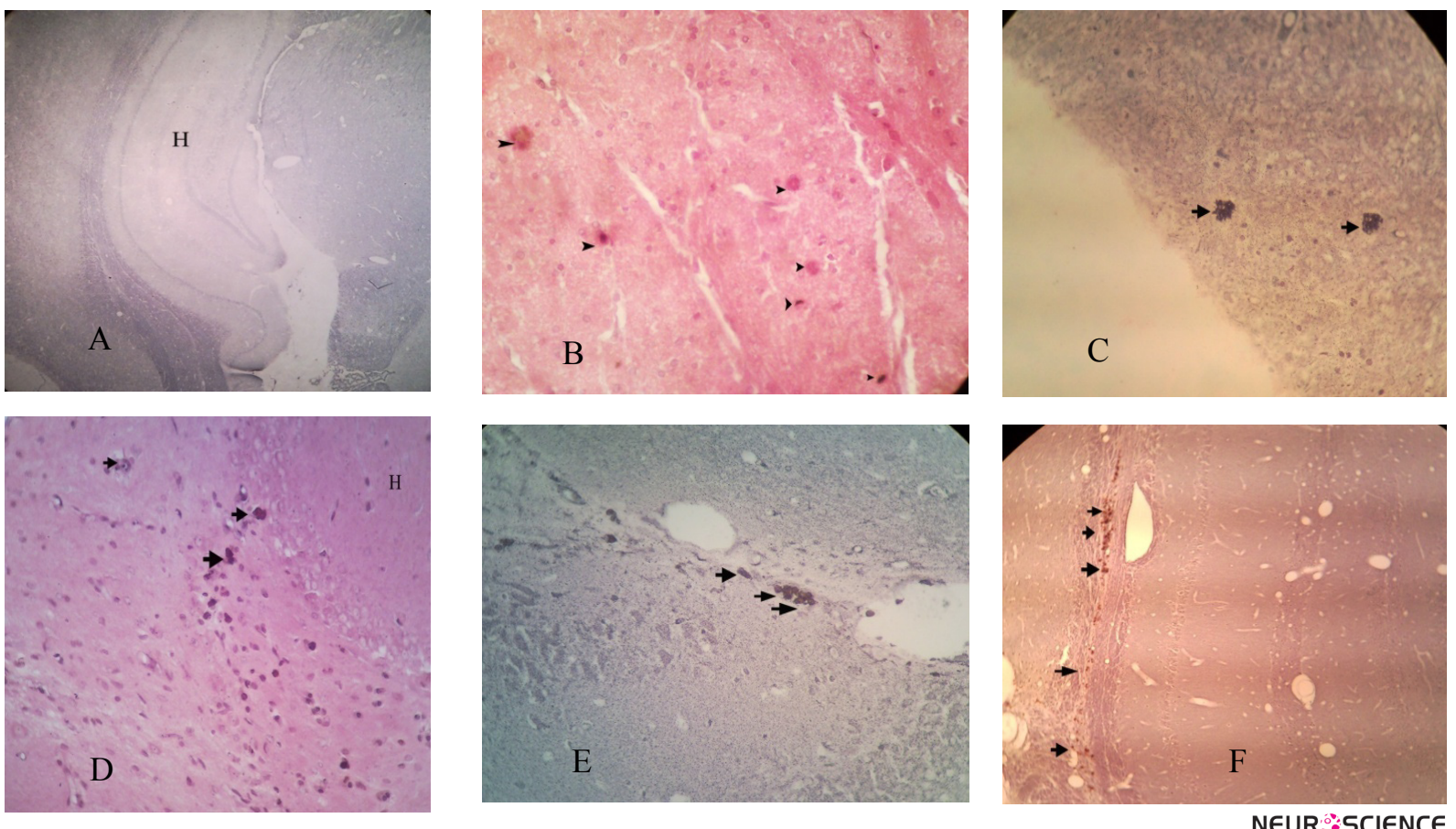

Figure 5. $A \beta$ plaques in CA1 region of hippocampus $(H)(\times 40)$. A) control group, $B) A D$ group, $C) A D+G A 50, D) A D+G A 100$, E) AD+GA200, F) AD+Veh (modified Bielschowsky staining). Arrows indicate the present level of AD plaques in CA1 area.

to disturbed glucose homeostasis due to increased insulin resistance and decreased beta-cell mass (Valizadeh, Eidi, Sarkaki, Farbood, \& Mortazavi, 2012; Park et al., 2013).

Although the exact cause of AD remains elusive, mounting evidence continues to support the involvement of neuro-inflammation in the development of AD. A study showed that the total number of intersection points of dendrites and spine density in hippocampal neurons in the $\mathrm{AD}$ model group decreased compared to the control group (Chu et al., 2014; Wan et al., 2014). So, these results indicate that anti-inflammatory and immunosuppressive agents can alleviate the degeneration of dendritic spines in hippocampal neurons in rats' model of AD.

Alzheimer disease (AD) pathology shows neuronal damage in special vulnerable brain regions and circuits involved in memory and language, namely the hippocampus and cerebral cortex, which appears to be preceded by synaptic and neuronal dysfunction. The relevance of synaptic mitochondria synapses are sites of extensive $\mathrm{Ca}^{2+}$ fluctuations since synaptic transmission requires high levels of ATP and constant regulation of intracellular $\mathrm{Ca} 2+$ concentration, enduring synaptic mitochondria vital for maintenance of synaptic function and transmission (Ferreiro et al., 2012).

Recent studies in mild cognitive impairment (MCI) and late-stage $\mathrm{AD}$ patients demonstrated a significant disease- dependent increase in oxidative markers localized mainly in the synapses. Interestingly, the levels of oxidative markers suggest the involvement of oxidative stress in AD-related synaptic loss ( Ansari \& Scheff, 2011)

Previous studies revealed that GA has anti-inflammatory and antioxidative effects to improve brain trauma after cerebral ischemia and traumatic brain injury. Current findings are consistent with previous studies (Mansouri et al., 2013; Korani et al., 2014; Naghizadeh \& Mansouri, 2014). In AD, synaptic dysfunction and loss of synapses are probably due to defects in synaptic mitochondria, which leads to alterations in cognitive function (Ansari \& Scheff, 2011), and interestingly, this seems to be related to reactive oxygen species (ROS) production and altered $\mathrm{Ca}^{2+}$ dynamics at the synapse (Kang et al., 2011; Guo, Guan, Huang, Wang, \& Shi, 2013).

In mouse hippocampal neurons, $A \beta$ was demonstrated to impair mitochondrial movements, reduce mitochondrial length, and cause synaptic degeneration (Calkins \& Reddy, 2011). Compared to nonsynaptic mitochondria, synaptic mitochondria showed a greater degree of age-dependent accumulation of $A \beta$ and mitochondrial alterations. The fact that synaptic mitochondria, especially A $\beta$-rich synaptic mitochondria, are more susceptible to $\mathrm{A} \beta$-induced damage in AD. Indeed, synaptic mitochondria are more sensitive to ROS than nonsynaptic mitochondria (Ferreiro et al., 2012). 
$\mathrm{A} \beta$ is a potent neurotoxic peptide and has a pivotal role in cognitive deficit and reduced synaptic plasticity in AD. Administration of $A \beta_{1-42}$ drastically attenuated the LTP of DG neurons (Babri et al., 2014). In the current work, it was appeared the hippocampal LTP properties were decayed after $\mathrm{AD}$ induced by $\mathrm{A} \beta$, which is consistent with some findings by other investigators indicating that $A \beta$ can disrupt excitatory glutamatergic synaptic function at synaptic level by acute depression of basal glutamatergic synaptic transmission through both presynaptic and postsynaptic dysfunction (Yao, Zou, Sun, \& Ren, 2013).

In $\mathrm{AD}$, synapses are the primary sites of $\mathrm{Ca}^{2+}$ deregulation due to over activation of glutamate receptors. These receptors are concentrated on postsynaptic spines of neuronal dendrites where particularly subjected to high levels of $\mathrm{Ca}^{2+}$ influx, oxidative stress, and ATP demand (Dalton, Wu, Wang, Floresco, \& Phillips 2012). In fact, $\mathrm{Ca} 2+$ influx through NMDA receptors (NMDARs) induced by synaptic activity is required for many types of synaptic plasticity and underlies some forms of learning and memory. Thus, glutamate receptors are likely sites at which neurodegenerative processes are initiated in aging and early $\mathrm{AD}$, playing an important role in decreased synaptic function (Gholamipour-Badie, Naderi, Khodagholi, Shaerzadeh, \& Motamedi, 2013).

In previous studies we have shown that oral administration of different doses of GA after induction of animal models of neurodegenerative diseases such as PD and cerebral ischemia improved the cognitive behavior and brain electrophysiology deficits through changes in the pro -and anti- inflammatory cytokines levels, brain edema, antioxidative effects in brain tissue as well as improving the blood brain barrier permeability (Farbood et al., 2015; Mansouri et al., 2013). These findings consistent with other investigations showed the potential therapeutic effect of antioxidants to protect neurons against $A \beta$ induced cell death and lipid peroxidation. These natural antioxidants have been found to improve cognitive function in aged rats and prevent learning and memory deficits following brain injury (Mansouri et al., 2014; Farbood et al., 2015). Clinical studies have also described positive effects of antioxidant treatments in AD, and treatment with antioxidants has been reported to slow the progression of AD (McDaid et al., 2005; Scuderi et al., 2014).

Various pharmacological activities of GA such as anticancer (Yang et al., 2006) and antioxidant function (Yang et al., 2006) have been reported. This compound has also been described as an excellent free radical scavenger (Isuzugawa, Inoue, \& Ogihara, 2001). Several line of studies demonstrated that administration of GA could improve the cognitive deficits after cerebral damages in rats. Cognitive enhancing and neuroprotective effect of GA are associated with the antioxidant of this compound (Korani et al., 2014).

GA effectively decreased the brain level of the ROS, indicating that GA exerts antioxidative activity partially by modulating brain dysfunctions (Sun et al., 2014a).

Ki-Yeon Yoo and his colleagues (2010) reported that oral administration of epigallocatechin-3-gallate (EGCG), a major catechin of green tea can promote cell proliferation, neuronal fates, neuroblast differentiation and maturation of neurons in the hippocampal dentate gyrus. It has also been reported that the chronic administration of EGCG improves learning ability in rats and mice. In addition, when mouse hippocampal slices were pretreated with EGCG for 1 hour prior to experiment, the level of highfrequency stimulation-evoked LTP increased significantly during synaptic transmission between the hippocampal regions. These results suggested that oral administration of EGCG can enhance cell proliferation and increase the number of neuroblasts in mice hippocampal dentate gyrus (Yoo et al., 2010). Our finding in the current work showed that treatment of $\mathrm{AD}$ rats with GA improves brain electrical activity, which is consistent with the previous results.

In this study, we found no significant difference in evoked field potentials between sham operated and sham group received GA for the same manner. This study revealed that GA improves only the synaptic failure induced by $A \beta$ peptide and can be introduced as a promising multipotent pharmacological agent in the prevention or treatment of $\mathrm{AD}$ in the future.

Our finding showed that $\mathrm{A} \beta$ disrupted synaptic plasticity in hippocampus due to constituent of the senile plaques and neuronal apoptosis. Treatment of AD (but not healthy) rats with GA improved brain histology and electrophysiology damages as dose dependent. Furthermore, our findings might raise a possibility of therapeutic applications of GA for preventing and or treating neurodegenerative diseases.

\section{Acknowledgments}

This research was carried out in Ahvaz Physiology Research Center (APRC-73). It extracted as part of Zohreh Valizadeh's PhD thesis. Authors thank the research affairs of Ahvaz Jundishapur University of Medical Sciences as well as main animal house staff. 


\section{References}

Ansari, M. A., \& Scheff, S. W. (2011). NADPH-oxidase activation and cognition in Alzheimer's disease progression. Free Radical Biology \& Medicine, 51(1), 171-178.

Babri, S., Mohaddes, G., Feizi, I., Mohammadnia, A., Niapour, A., Ali-Hemmati, A., et al. (2014). Effect of troxerutin on synaptic plasticity of hippocampal dentate gyrus neurons in a beta-amyloid model of Alzheimers disease: an electrophysiological study. European Journal of Pharmacology, 732, 19-25.

Calkins, M. J., \& Reddy, P. H. (2011). Amyloid beta impairs mitochondrial anterograde transport and degenerates synapses in Alzheimer's disease neurons. Biochimica et Biophysica Acta, 1812(4), 507-513.

Chen, D. L., Zhang, P., Lin, L., Zhang, H. M., Deng, S. D., Wu, Z. Q., et al. (2014). Protective effects of bajijiasu in a rat model of Abeta(2)(5)(-)(3)(5)-induced neurotoxicity. Journal of Ethnopharmacology, 154(1), 206-217.

Chu, S., Gu, J., Feng, L., Liu, J., Zhang, M., Jia, X., et al. (2014). Ginsenoside Rg5 improves cognitive dysfunction and betaamyloid deposition in STZ-induced memory impaired rats via attenuating neuroinflammatory responses. International Immunopharmacology, 19(2), 317-326.

Dalton, G. L., Wu, D. C., Wang, Y. T., Floresco, S. B., \& Phillips, A. G. (2012). NMDA GluN2A and GluN2B receptors play separate roles in the induction of LTP and LTD in the amygdala and in the acquisition and extinction of conditioned fear. Neuropharmacology, 62(2), 797-806.

Ehrlich, D., Hochstrasser, T., \& Humpel, C. (2013). Effects of oxidative stress on amyloid precursor protein processing in rat and human platelets. Platelets, 24(1), 26-36.

Engelhart, M. J., Geerlings, M. I., Ruitenberg, A., van Swieten, J. C., Hofman, A., Witteman, J. C., et al. (2002). Dietary intake of antioxidants and risk of Alzheimer's disease. Journal of the American Medical Association, 287(24), 3223-3229.

Engelhart, Y., Sarkaki, A., Dianat, M., Khodadadi, A., Haddad, M. K., \& Mashhadi-Zadeh, S. (2015). Ellagic acid prevents cognitive and hippocampal long-term potentiation deficits and brain inflammation in rat with traumatic brain injury. Life Sciences, 124, 120-127.

Farbood, Y., Sarkaki, A., Hashemi, S., Mansouri, M. T., \& Dianat, M. (2013). The effects of gallic acid on pain and memory following transient global ischemia/reperfusion in Wistar rats. Avicenna Journal of Phytomedicine, 3(4), 329-340.

Ferihan, C., \& Sibel, D. (2006). The effect of interahippocampal beta amyloid (1-42) peptide injection on oxidant and antioxdidant status in rat brain. Annals of the New York Academy of Sciences, 1100(1), 8 .

Ferreiro, E., Baldeiras, I., Ferreira, I. L., Costa, R. O., Rego, A. C., Pereira, C. F., et al. (2012). Mitochondrial- and endoplasmic reticulum-associated oxidative stress in Alzheimer's disease: from pathogenesis to biomarkers. International Journal of Cell Biology, 2012, 735206 .

Ferruzzi, M. G., Lobo, J. K., Janle, E. M., Cooper, B., Simon, J. E., Wu, Q. L., et al. (2009). Bioavailability of gallic acid and catechins from grape seed polyphenol extract is improved by repeated dosing in rats: implications for treatment in Alzheimer's disease. Journal of Alzheimer's Disease, 18(1), 113-124.
Gholamipour-Badie, H., Naderi, N., Khodagholi, F., Shaerzadeh, F., \& Motamedi, F. (2013). L-type calcium channel blockade alleviates molecular and reversal spatial learning and memory alterations induced by entorhinal amyloid pathology in rats. Behavioural Brain Research, 237, 190-199.

Goryacheva, A. V., Kruglov, S. V., Pshennikova, M. G., Smirin, B. V., Malyshev, I. Y., Barskov, I. V., et al. (2010). Adaptation to intermittent hypoxia restricts nitric oxide overproduction and prevents beta-amyloid toxicity in rat brain. Nitric Oxide: Biology and Chemistry, 23(4), 289-299.

Guo, L. L., Guan, Z. Z., Huang, Y., Wang, Y. L., \& Shi, J. S. (2013) The neurotoxicity of beta-amyloid peptide toward rat brain is associated with enhanced oxidative stress, inflammation and apoptosis, all of which can be attenuated by scutellarin. Experimental and Toxicologic Pathology, 65(5), 579-584.

Gureviciene, I., Ikonen, S., Gurevicius, K., Sarkaki, A., van Groen, T., Pussinen, R., et al. (2004). Normal induction but accelerated decay of LTP in APP + PS1 transgenic mice. Neurobiology of Disease, 15(2), 188-195.

Hartman, R. E., Shah, A., Fagan, A. M., Schwetye, K. E., Parsadanian, M., Schulman, R. N., et al. (2006). Pomegranate juice decreases amyloid load and improves behavior in a mouse model of Alzheimer's disease. Neurobiology of Disease, 24(3), 506-515.

Hochstrasser, T., Hohsfield, L. A., Sperner-Unterweger, B., \& Humpel, C. (2013). Beta-Amyloid induced effects on cholinergic, serotonergic, and dopaminergic neurons is differentially counteracted by anti-inflammatory drugs. Journal of Neuroscience Research, 91(1), 83-94.

Isuzugawa, K., Inoue, M., \& Ogihara, Y. (2001). Catalase contents in cells determine sensitivity to the apoptosis inducer gallic acid. Biological and Pharmaceutical Bulletin, 24(9), 10221026.

Joseph, J. A., Shukitt-Hale, B., \& Casadesus, G. (2005). Reversing the deleterious effects of aging on neuronal communication and behaviour: beneficial properties of fruit polyphenolic compounds. American Journal of Clinical Nutrition, 81(1), 313S$316 S$.

Kang, D. E., Roh, S. E., Woo, J. A., Liu, T., Bu, J. H., Jung, A. R., et al. (2011). The interface between cytoskeletal aberrations and mitochondrial dysfunction in Alzheimer's disease and related disorders. Experimental Neurobiology, 20(2), 67-80.

Kim, J. H., Anwyl, R., Suh, Y. H., Djamgoz, M. B., \& Rowan, M. J (2001). Use-dependent effects of amyloidogenic fragments of (beta)-amyloid precursor protein on synaptic plasticity in rat hippocampus in vivo. The Journal of Neuroscience, 21(4), 13271333.

Korani, M. S., Farbood, Y., Sarkaki, A., Fathi-Moghaddam, H., \& Mansouri, M. T. (2014). Protective effects of gallic acid against chronic cerebral hypoperfusion-induced cognitive deficit and brain oxidative damage in rats. European Journal of Pharmacology, 733, 62-67.

Kostrzewa, R. M., \& Segura-Aguilar, J. (2003). Novel mechanisms and approaches in the study of neurodegeneration and neuroprotection. A review. Neurotoxicity Research, 5(6), 375 383.

Kowall, N. W., Beal, M. F., Busciglio, J., Duffy, L. K., \& Yankner B. A. (1991). An in vivo model for the neurodegenerative effects of beta amyloid and protection by substance P. Proceed- 
ings of the National Academy of Sciences of the United States of America, 88(16), 7247-7251.

Kratz, J. M., Andrighetti-Frohner, C. R., Kolling, D. J., Leal, P. C., Cirne-Santos, C. C., Yunes, R. A., et al. (2008a). Anti-HSV-1 and anti-HIV-1 activity of gallic acid and pentyl gallate. Memórias do Instituto Oswaldo Cruz, 103(5), 437-442.

Kratz, J. M., Andrighetti-Frohner, C. R., Leal, P. C., Nunes, R. J., Yunes, R. A., Trybala, E., et al. (2008b). Evaluation of antiHSV-2 activity of gallic acid and pentyl gallate. Biological $\mathcal{E}$ Pharmaceutical Bulletin, 31(5), 903-907.

Mansouri, M. T., Naghi-Zadeh, B., Ghorban-Zadeh, B., Farbood, Y., Sarkaki, A., \& Bavarsad, K. (2013). Gallic acid prevents memory deficits and oxidative stress induced by intracerebroventricular injection of streptozotocin in rats. Pharmacology Biochemistry and Behavior, 111, 90-96.

Mansouri, M. T., Soltani, M., Naghi-Zadeh, B., Farbood, Y., Mashak, A., \& Sarkaki, A. (2014). A possible mechanism for the anxiolytic-like effect of gallic acid in the rat elevated plus maze. Pharmacology Biochemistry and Behavior, 117, 40-46.

McDaid, D. G., Kim, E. M., Reid, R. E., Leslie, J. C., Cleary, J., \& O'Hare, E. (2005). Parenteral antioxidant treatment preserves temporal discrimination following intrahippocampal aggregated Abeta (1-42) injections. Behavioural Pharmacology, 16(4), 237-242.

Naghizadeh, B., \& Mansouri, M. T. (2014). Protective effects of gallic acid against Streptozotocin-induced oxidative damage in rat striatum. Drug Research, 65(10):515-20.

Niho, N., Shibutani, M., Tamura, T., Toyoda, K., Uneyama, C., Takahashi, N., et al. (2001). Subchronic toxicity study of gallic acid by oral administration in F344 rats. Food and Chemical Toxicology, 39(11), 1063-1070.

Nobakht, M., Hoseini, S. M., Mortazavi, P., Sohrabi, I., Esmailzade, B., Rahbar Rooshandel, N., et al. (2011). Neuropathological changes in brain cortex and hippocampus in a rat model of Alzheimer's disease. Iranian Biomedical Journal, $15(1-2), 51-58$

Park, S., Kim da, S., Kang, S., \& Moon, N. R. (2013). Beta-Amyloid-induced cognitive dysfunction impairs glucose homeostasis by increasing insulin resistance and decreasing beta-cell mass in non-diabetic and diabetic rats. Metabolism: Clinical and Experimental, 62(12), 1749-1760.

Paxinos, G., \& Waston, C. (2006). The rat brain stereotaxic coordinates: Hard Cover Edition (6 ${ }^{\text {th }}$ Ed.). San Diego, Elsevier: Academic Press.

Qi, F. H., Jing, T. Z., Wang, Z. X., \& Zhan, Y. G. (2009). Fungal endophytes from Acer ginnala Maxim: isolation, identification and their yield of gallic acid. Letters in Applied Microbiology, 49(1), 98-104.

Scuderi, C., Stecca, C., Bronzuoli, M. R., Rotili, D., Valente, S., Mai, A., et al. (2014). Sirtuin modulators control reactive gliosis in an in vitro model of Alzheimer's disease. Frontiers in Pharmacology, 5, 89 .

Shukitt-Hale, B., Carey, A., Simon, L., Mark, D. A., \& Joseph, J. A. (2006). Effects of concord grape juice on cognitive and motor deficits in aging. Nutrition, 22(3), 295-302.
Sladjana, A. (2011). A prediction survival model for out-of-hospital cardiopulmonary resuscitations. Journal of Critical Care, 26(2), e211-228.

Sun, J., Li, Y. Z., Ding, Y. H., Wang, J., Geng, J., Yang, H., et al. (2014a). Neuroprotective effects of gallic acid against hypoxia/reoxygenation-induced mitochondrial dysfunctions in vitro and cerebral ischemia/reperfusion injury in vivo. Brain Research, 1589, 126-139.

Sun, Q., Jia, N., Wang, W., Jin, H., Xu, J., \& Hu, H. (2014b). Activation of SIRT1 by curcumin blocks the neurotoxicity of amyloid-beta25-35 in rat cortical neurons. Biochemical and Biophysical Research Communications, 448(1), 89-94.

Valizadeh, Z., Eidi, A., Sarkaki, A., Farbood, Y., \& Mortazavi. P. (2012). Dementia type of Alzheimer's disease due to beta-Amyloid was improved by gallic acid in rats. HealthMED Journal, 6(11), 3648-3656.

Wan, B., Hu, X., Nie, J., Zhou, M., Yang, B., \& Li, Y., et al. (2014). Effects of triptolide on degeneration of dendritic spines induced by Abeta1-40 injection in rat hippocampus. Neurological Sciences, 35(1), 35-40.

Wen, P., Luo, H., Zhou, L., Song, Z., Li, W., \& Zhou, J. (2014) Effects of tanshinone IIA on the expressions of caspase-3, Akt and NF-kappaB in the brains of rat models of Alzheimer's disease. Xi bao yu fen zi mian yi xue za zhi (Chinese Journal of Cellular and Molecular Immunology), 30(2), 155-159.

Yang, H. L., Chang, W. H., Chia, Y. C., Huang, C. J., Lu, F. J. Hsu, H. K., et al. (2006). Toona sinensis extracts induces apoptosis via reactive oxygen species in human premyelocytic leukemia cells. Food and Chemical Toxicology, 44(12), 1978-1988.

Yao, W., Zou, H. J., Sun, D., \& Ren, S. Q. (2013). Abeta induces acute depression of excitatory glutamatergic synaptic transmission through distinct phosphatase-dependent mechanisms in rat CA1 pyramidal neurons. Brain Research, 1515, 88-97.

Yoo, K. Y., Choi, J. H., Hwang, I. K., Lee, C. H., Lee, S. O., Han, S. M., et al. (2010). (-)-Epigallocatechin-3-gallate increases cell proliferation and neuroblasts in the subgranular zone of the dentate gyrus in adult mice. Phytotherapy Research, 24(7), 10651070

Zhan, X. Q., Yao, J. J., Liu, D. D., Ma, Q., \& Mei, Y. A. (2014). Abeta 40 modulates GABA(A) receptor alpha6 subunit expression and rat cerebellar granule neuron maturation through the ERK/mTOR pathway. Journal of Neurochemistry, 128(3) 350-362.

Zhang, J., Zhen, Y. F., Pu Bu Ci, R., Song, L. G., Kong, W. N. Shao, T. M., et al. (2013). Salidroside attenuates beta amyloidinduced cognitive deficits via modulating oxidative stress and inflammatory mediators in rat hippocampus. Behavioural Brain Research, 244, 70-81. 